\title{
Controllability of a stochastic functional differential equation driven by a fractional Brownian motion
}

Jingqi $\operatorname{Han}^{1}$ and Litan Yan²*

"Correspondence:

litan-yan@hotmail.com

2Department of Mathematics,

College of Science, Donghua

University, Shanghai, P.R. China

Full list of author information is

available at the end of the article

\section{Springer}

\begin{abstract}
Let $U, V$ and $W$ be three Hilbert spaces and let $B^{H}$ be a $W$-valued fractional Brownian motion with Hurst index $H \in\left(\frac{1}{2}, 1\right)$. In this paper, we consider the approximate controllability of the Sobolev-type fractional stochastic differential equation

$$
\begin{cases}{ }^{c} D_{t}^{\alpha}[L x(t)]=A x(t)+f\left(t, x_{t}\right)+B u(t)+\sigma(t) \frac{d}{d t} B^{H}(t), & t \in(0, T], \\ x(t)=\phi(t), & t \in(-\infty, 0],\end{cases}
$$

where ${ }^{\mathrm{c}} D^{\alpha}$ is the Caputo fractional derivative of order $\alpha \in(1-H, 1)$, the time history $x_{t}:(-\infty, 0] \rightarrow x_{t}(\theta)=x(t+\theta)$ with $t>0$ belonging to the phase space $\mathscr{B}_{h}$, the control function $u(\cdot)$ is given in $L^{2}([0, T], V), B$ is a bounded linear operator from $V$ into $U$. Under some suitable conditions, we show that the system is approximately controllable on $[0, T]$ and we give an example to illustrate the theory.
\end{abstract}

MSC: $60 \mathrm{G} 22 ; 60 \mathrm{H} 05 ; 60 \mathrm{H} 10$

Keywords: Fractional stochastic functional differential equation; Fractional Brownian motion; Fractional calculus; Controllability

\section{Introduction}

The study of controllability is one of the important parts of mathematical control theory in both deterministic and stochastic control theory. There are lots of publications working on control problems of various systems [1-4]. Complete controllability means the systems can be steered to arbitrary final state while the systems with approximate controllability just can be steered to a small neighborhood of the final state. Even though the concept of the approximate controllability is weaker than the complete controllability, it is prevalent to consider approximate controllable systems which can be adequate in application [5-7]. In fact, the approximate controllability of systems represented by nonlinear evolution equations has been studied by several authors. In [8], Sakthivel et al. studied a class of control systems governed by the semilinear fractional differential equations in Hilbert spaces by using the semigroup theory, the fractional power theory and fixed point theorem. Fu and Mei [9] investigated the approximate controllability of semilinear neutral functions differential systems with finite delay.

(c) The Author(s) 2018. This article is distributed under the terms of the Creative Commons Attribution 4.0 International License (http://creativecommons.org/licenses/by/4.0/), which permits unrestricted use, distribution, and reproduction in any medium, provided you give appropriate credit to the original author(s) and the source, provide a link to the Creative Commons license, and indicate if changes were made. 
Due to the extensive applications in various fields such as science and engineering, fractional differential equations attract more and more attention of experts and scholars. Fractional differential equations may be derived from the particle sticking and trapping phenomena which would be more accurate to describe certain physical phenomena (see, for examples, [10-12]). In addition, Sobolev-type equation appears in all kinds of physical problems such as flow of fluid through fissured rocks, thermodynamics, propagation of long waves of small amplitude (see [13]). Therefore, it is necessary and significative to study fractional order differential equations of Sobolev-type (see [14, 15] and the references therein). The existence and uniqueness of mild solution to Sobolev-type fractional nonlocal dynamical equations in Banach spaces is shown in [16]. By using the fractional calculus, semigroup theory and stochastic analysis techniques, [17] considered a class of nonlinear fractional Sobolev-type stochastic differential equations in a Hilbert space.

On the other hand, the property of long memory is widely used in describing the phenomena in fields like hydrology and geophysics as well as economics and telecommunications. As an extension of Brownian motion, fractional Brownian motion ( $\mathrm{fBm})$ is a selfsimilar Gaussian processes which have the properties of long/short-range dependence. However, in comparison with Brownian motion, the process is neither a semi-martingale nor a Markov process. For this reason, there are a few publications leaning the systems which are driven by this type of perturbation. In [18], the authors first studied the fractional Brownian motion in Hilbert spaces and some related stochastic equations. We refer to $[19,20]$ and the references therein for the details of the theory of stochastic calculus for fractional Brownian motion. However, it should be emphasized that to the best of our knowledge the controllability of stochastic functional differential equation of Sobolevtype driven by fractional Brownian motion has not been studied yet and the aim of this paper is to do some further research on this problem.

Motivated by these results, in this paper we study the approximate controllability of the Sobolev-type fractional stochastic differential equations of the form

$$
\begin{cases}{ }^{\mathrm{c}} D_{t}^{\alpha}[L x(t)]=A x(t)+f\left(t, x_{t}\right)+B u(t)+\sigma(t) \frac{d}{d t} B^{H}(t), & t \in(0, T], \\ x(t)=\phi(t), & t \in(-\infty, 0] .\end{cases}
$$

In the above system, we assume that

- ${ }^{\mathrm{c}} D^{\alpha}$ is the Caputo fractional derivative of order $\alpha \in(1-H, 1)$,

- $A, L$ are two linear bounded operators on a Hilbert space $U$,

- $B$ is a bounded linear operator from the Hilbert space $V$ into Hilbert space $U$,

- the time history $x_{t}(\theta)=x(t+\theta), t>0$,

- $u(\cdot)$ is a control function on $L^{2}([0, T], V)$,

- $B^{H}=\left\{B^{H}(t), t \in[0, T]\right\}$ is a cylindrical fractional Brownian motion with Hurst index $H \in\left(\frac{1}{2}, 1\right)$

- the functions $f$ and $\sigma$ are two Borel functions with some suitable conditions.

The paper is organized as follows. In Sect. 2, we represent some preliminaries for stochastic integral of fractional Brownian motion in Hilbert space. In Sect. 3, we obtain the approximate controllability results of the Sobolev-type fractional stochastic system (1.1). In Sect. 4, we give an example as an application. 


\section{Preliminaries}

In this section, we will introduce some definitions, lemmas and notions which will be used in the next section.

\subsection{Fractional Brownian motion}

Let $\left(\Omega, \mathscr{F},\left(\mathscr{F}_{t}\right)\right.$, P) be a complete filtered probability space. A fractional Brownian motion (fBm) $\beta^{H}=\left\{\beta^{H}(t), t \in[0, T]\right\}$ with Hurst index $H \in(0,1)$ is a mean zero Gaussian process such that $\beta^{H}(0)=0$ and

$$
\mathrm{E}\left(\beta^{H}(t) \beta^{H}(s)\right)=\frac{1}{2}\left(s^{2 H}+t^{2 H}-|t-s|^{2 H}\right)
$$

for all $t, s \geq 0$. When $H=1 / 2, \beta^{H}$ coincides with the standard Brownian motion, and when $H \neq \frac{1}{2}$ it is neither a semi-martingale nor a Markov process. The $\mathrm{fBm} \beta^{H}$ admits the following integral representation:

$$
\beta^{H}(t)=\int_{0}^{t} K_{H}(t, s) d W(t)
$$

for all $t \geq 0$, where $\{W(t), 0 \leq t \leq T\}$ is a standard Brownian motion and the kernel $K_{H}(t, s)$ satisfies

$$
\frac{\partial K_{H}}{\partial t}(t, s)=\kappa_{H}\left(H-\frac{1}{2}\right)\left(\frac{s}{t}\right)^{\frac{1}{2}-H}(t-s)^{H-\frac{3}{2}}
$$

with a normalizing constant $\kappa_{H}>0$ such that $E\left(\beta_{1}^{H}\right)^{2}=1$. Throughout this paper we assume that $\frac{1}{2} \leq H<1$ is arbitrary but fixed.

Let $\mathcal{H}$ be the completion of the linear space $\mathcal{E}$ generated by the indicator functions $1_{[0, t]}$, $t \in[0, T]$ with respect to the inner product

$$
\left\langle 1_{[0, s]}, 1_{[0, t]}\right\rangle_{\mathcal{H}}=\frac{1}{2}\left(t^{2 H}+s^{2 H}-|t-s|^{2 H}\right) .
$$

The mapping

$$
\mathcal{E} \ni \varphi \rightarrow \beta^{H}(\varphi):=\int_{0}^{T} \varphi(s) d \beta^{H}(s)
$$

is an isometry from $\mathcal{E}$ to the Gaussian space generated by $\beta^{H}$ and it can be extended to $\mathcal{H}$, which is called the Wiener integral with respect to $\beta^{H}$. Consider the operator $K_{H}^{*}$ from $\mathcal{E}$ to $L^{2}([0, T])$ defined by

$$
\left(K_{H}^{*} \varphi\right)(s)=\int_{s}^{T} \varphi(t) \frac{\partial K_{H}}{\partial t}(t, s) d t
$$

for $\varphi \in \mathcal{E}$. Then the operator $K_{H}^{*}$ is an isometry between $\mathcal{E}$ and $L^{2}([0, T])$ which can be also extended to the Hilbert space $\mathcal{H}$.

Lemma 2.1 For every $\varphi \in \mathcal{H}$, we have

$$
\int_{0}^{T} \varphi(s) d \beta^{H}(s)=\int_{0}^{T}\left(K_{H}^{*} \varphi\right)(s) d W(s) .
$$


We now recall that the definition of stochastic integral of $\mathrm{fBm}$ in the Hilbert space $V$. Let $\left\{B^{H}(t), 0 \leq t \leq T\right\}$ be a $W$-valued $\mathscr{F}_{t}$-adapted fBm defined on $\left(\Omega, \mathscr{F},\left(\mathscr{F}_{t}\right), \mathrm{P}\right)$ with the representation of the form

$$
B^{H}(t)=\sum_{n=1}^{\infty} \sqrt{\lambda_{n}} \beta_{n}^{H}(t) e_{n}, \quad t \geq 0
$$

where $\left\{e_{n}\right\}_{n \in \mathbb{N}}$ is a complete orthogonal basis in $W$, and

- $\left\{\beta_{n}^{H}, n=1,2, \ldots\right\}$ is a sequence of independent $\mathrm{fBms}$ with the same Hurst index $H \in\left(\frac{1}{2}, 1\right)$,

- $\left\{\lambda_{n} ; n \in \mathbb{N}\right\}$ is a bounded sequence of non-negative real numbers such that $Q e_{n}=\lambda_{n} e_{n}$,

- $Q$ is a non-negative self-adjoint trace class operator with finite trace

$$
\operatorname{Tr} Q=\sum_{n=1}^{\infty} \lambda_{n}<+\infty
$$

Let $\varphi:[0, T] \rightarrow L_{2}^{0}(W, U)$ such that

$$
\sum_{n=1}^{\infty}\left\|K_{H}^{*}\left(\varphi Q^{\frac{1}{2}} e_{n}\right)\right\|_{L_{2}^{0}([0, T] ; \zeta)}<\infty
$$

where $L_{2}^{0}(W, U)$ is the space of all Hilbert-Schmidt operators from $Q^{\frac{1}{2}} W$ to $U$ with norm $\|\cdot\|_{L_{2}^{0}(W, U)}$ defined by

$$
\|\xi\|_{L_{2}^{0}(W, U)}=\left\|\xi Q^{\frac{1}{2}}\right\|_{H-S}^{2}=\operatorname{Tr}\left(\xi Q \xi^{*}\right)=\sum_{n=1}^{\infty}\left\|\sqrt{\lambda_{n}} \xi e_{n}\right\|^{2}
$$

Definition 2.1 Let $\varphi:[0, T] \rightarrow L_{2}^{0}(W, U)$ satisfy (2.1). We define the stochastic integral $\int_{0}^{t} \varphi(s) d B^{H}(s)$ by

$$
\begin{aligned}
\int_{0}^{t} \varphi(s) d B^{H}(s) & :=\sum_{n=1}^{\infty} \int_{0}^{t} \varphi(s) Q^{\frac{1}{2}} e_{n} d \beta_{n}^{H} \\
& =\sum_{n=1}^{\infty} \int_{0}^{t}\left(K_{H}^{*}\left(\varphi Q^{\frac{1}{2}} e_{n}\right)\right)(s) d B(s) .
\end{aligned}
$$

Lemma 2.2 Let $\varphi:[0, T] \rightarrow L_{2}^{0}(W, U)$ satisfy (2.1). Then, for any $a, b \in[0, T]$ with $a<b$ we have

$$
\mathrm{E}\left\|\int_{a}^{b} \varphi(s) d B^{H}(s)\right\|^{2} \leq c H(2 H-1)(b-a)^{2 H-1} \sum_{n=1}^{\infty} \int_{a}^{b}\left\|\varphi(s) Q^{\frac{1}{2}} e_{n}\right\|^{2} d s .
$$

In addition, $\sum_{n=1}^{\infty}\left\|\varphi(s) Q^{\frac{1}{2}} e_{n}\right\|$ is uniformly convergent in $t \in[0, T]$, then we have

$$
\mathrm{E}\left\|\int_{a}^{b} \varphi(s) d B^{H}(s)\right\|^{2} \leq c H(2 H-1)(b-a)^{2 H-1} \sum_{n=1}^{\infty} \int_{a}^{b}\|\varphi(s)\|_{L_{2}^{0}(W, U)}^{2} d s .
$$




\subsection{Some assumptions}

In this subsection, we recall that some notions of fractional calculus and give some assumptions for the stochastic system (1.1). Recall that the fractional integral $I^{\alpha}$ of order $\alpha$ for a function $f:[0, \infty) \rightarrow \mathbb{R}$ is defined as

$$
I^{\alpha} f(t)=\frac{1}{\Gamma(\alpha)} \int_{0}^{t} \frac{f(s)}{(t-s)^{1-\alpha}} d s, \quad t>0, \alpha>0
$$

provided the right side is point-wise defined on $[0, \infty)$, where $\Gamma(\cdot)$ is the gamma function, which is defined by $\Gamma(x):=\int_{0}^{\infty} t^{x-1} e^{-t} d t$. Moreover, the Caputo derivative ${ }^{\mathrm{c}} D^{\alpha}$ of order $\alpha$ for a function $f \in \mathbf{C}^{n}([0, \infty))$ is defined as

$$
{ }^{\mathrm{c}} D_{t}^{\alpha} f(t)=\frac{1}{\Gamma(n-\alpha)} \int_{0}^{t} \frac{f^{(n)}(s)}{(t-s)^{1+\alpha-n}} d s=I^{n-\alpha} f^{(n)}(t), \quad t>0, n-1<\alpha<n .
$$

If $f$ is an abstract function with values in $U$, then the integrals appearing in the above definitions are taken in Bochner's sense.

To study the stochastic system (1.1), we need some assumptions. Throughout this paper we assume that $U, V, W$ is three real separable Hilbert spaces with inner products $\langle\cdot, \cdot\rangle_{U}$, $\langle\cdot, \cdot\rangle_{V}$ and $\langle\cdot, \cdot\rangle_{W}$, respectively. We first give some conditions about the three operators $L$, $A, B$ as follows:

(A1) $A$ and $L$ are two linear unbounded operators on $U$ such that $D(A) \subset U, D(L) \subset U$, and $A$ is closed,

(A2) $D(L) \subset D(A)$,

$(\mathbb{A} 3) L^{-1}: U \rightarrow D(U)$ is compact,

(A4) $B$ is a bounded linear operator from $V$ into $U$.

Based on the above assumptions $(\mathbb{A} 1),(\mathbb{A} 2)$ and the closed graph theorem, the linear operator $A L^{-1}: U \rightarrow U$ the bounded, and $A L^{-1}$ generates a semigroup $\{S(t), t>0\}$ in $U$. Denote $M=\max _{t>0}\|S(t)\|,\|L\|=M_{1}$ and $\left\|L^{-1}\right\|=\widetilde{M}_{1}$.

For $x \in U$, we define two families $\left\{\mathscr{T}_{L}(t), t \geq 0\right\}$ and $\left\{\mathscr{S}_{L}(t), t \geq 0\right\}$ of operators by

$$
\mathscr{T}_{L}(t) x:=\int_{0}^{\infty} L^{-1} \xi_{\alpha}(\theta) S\left(t^{\alpha} \theta\right) x d \theta
$$

and

$$
\mathscr{S}_{L}(t) x:=\alpha \int_{0}^{\infty} L^{-1} \theta \xi_{\alpha}(\theta) S\left(t^{\alpha} \theta\right) x d \theta
$$

where

$$
\theta \mapsto \xi_{\alpha}(\theta)=\frac{1}{\pi \alpha} \sum_{n=1}^{\infty}(-\theta)^{n-1} \frac{1}{n !} \Gamma(n \alpha+1) \sin (n \pi \alpha)
$$

is a probability density function defined on $(0, \infty)$.

Lemma 2.3 ([21]) The operators $\mathscr{T}_{L}(t)$ and $\mathscr{S}_{L}(t)$ have the following properties: 
(i) For every $t \geq 0, \mathscr{T}_{L}(t)$ and $\mathscr{S}_{L}(t)$ are linear and bounded, and, moreover, for every $x \in U$

$$
\begin{aligned}
\left\|\mathscr{T}_{L}(t) x\right\| & \leq M \widetilde{M}_{1}\|x\|, \\
\left\|\mathscr{S}_{L}(t) x\right\| & \leq \frac{M \widetilde{M}_{1}}{\Gamma(\alpha)}\|x\| .
\end{aligned}
$$

(ii) $\mathscr{T}_{L}(t)$ and $\mathscr{S}_{L}(t)$ are strong continuous and compact.

We now introduce the abstract phase space. For a continuous function $h:(-\infty, 0] \rightarrow$ $(0, \infty)$ satisfying

$$
l:=\int_{-\infty}^{0} h(t) d t<\infty
$$

we define a phase space $\mathscr{B}_{h}$ associated with $h$ as follows (see, Cui and Yan [11]):

$$
\begin{aligned}
\mathscr{B}_{h}= & \left\{\phi:(-\infty, 0] \rightarrow U, \text { for any } a>0,\left(\mathrm{E}\|\phi(\theta)\|^{2}\right)^{1 / 2}\right. \text { is bounded } \\
& \text { and measurable functions on }[-a, 0] \text { with } \phi(0)=0 \\
& \text { and } \left.\int_{-\infty}^{0} h(s) \sup _{s \leq \theta \leq 0}\left(\mathrm{E}\|\phi(\theta)\|^{2}\right)^{1 / 2} d s<\infty\right\} .
\end{aligned}
$$

Clearly, $\left(\mathscr{B}_{h},\|\cdot\|_{\mathscr{B}_{h}}\right)$ is a Banach space if $\mathscr{B}_{h}$ is endowed with the norm

$$
\|\phi\|_{\mathscr{B}_{h}}=\int_{-\infty}^{0} h(s) \sup _{s \leq \theta \leq 0}\left(\mathrm{E}\|\phi(\theta)\|^{2}\right)^{1 / 2} d s
$$

for $\phi \in \mathscr{B}_{h}$. According to the definition of the fractional derivative and Caputo derivative, we rewrite (1.1) as the equivalent integral equation

$$
\begin{aligned}
L x(t)= & \phi(0)+\frac{1}{\Gamma(\alpha)} \int_{0}^{t}(t-s)^{\alpha-1}\left[A x(s)+f\left(s, x_{s}\right)+B u(s)\right] d s \\
& +\frac{1}{\Gamma(\alpha)} \int_{0}^{t}(t-s)^{\alpha-1} \sigma(s) d B^{H}(s) .
\end{aligned}
$$

We present the definition of mild solutions of (1.1).

Definition 2.2 An $U$-valued stochastic process $\{x(t), t \in[0, T]\}$ is a mild solution of (1.1) if the next conditions hold:

(i) $x(t)$ is measurable and $\mathscr{F}_{t}$-adapted, and $x_{t}$ is $\mathscr{B}_{h}$-valued,

(ii) for each $t \in[0, T], x(t)$ satisfies the equation

$$
\begin{aligned}
x(t)= & \mathscr{T}_{L}(t)(L \phi(0))+\int_{0}^{t}(t-s)^{\alpha-1} \mathscr{S}_{L}(t-s)\left[f\left(s, x_{s}\right)+B u(s)\right] d s \\
& +\int_{0}^{t}(t-s)^{\alpha-1} \mathscr{S}_{L}(t-s) \sigma(s) d B^{H}(s),
\end{aligned}
$$

(iii) $x(t)=\phi(t)$ on $(-\infty, 0]$ such that $\|\phi\|_{\mathscr{B}_{h}}^{2}<\infty$. 
Finally, in order to prove our main statement, we need some conditions on the functions $f$ and $\sigma$ as follows.

$(\mathbb{B} 1)$ Let the function $f:[0, T] \times \mathscr{B}_{h} \rightarrow U$ is continuous and there exist some constants $N_{f}>0, k_{f}>0$ such that, for $t \in[0, T]$ and $\xi, \eta \in \mathscr{B}_{h}$

$$
\mathrm{E}\|f(t, \xi)-f(t, \eta)\|^{2} \leq N_{f}\|\xi-\eta\|_{\mathscr{B}_{h}}
$$

for all $t \in[0, T]$ and $k_{f}=\sup _{t \in[0, T]}\|f(t, 0)\|^{2}$.

$(\mathbb{B} 2)$ For the complete orthogonal basis $\left\{e_{n}\right\}_{n \in \mathbb{N}}$ in $W$, the function $\sigma:[0, T] \rightarrow L_{2}^{0}(W, U)$ satisfy

$$
\sum_{n=1}^{\infty}\left\|\sigma Q^{\frac{1}{2}} e_{n}\right\|_{L^{2}([0, T], U)}<\infty
$$

and $\sum_{n=1}^{\infty}\left\|\sigma(t) Q^{\frac{1}{2}} e_{n}\right\|$ is uniformly convergent in $t \in[0, T]$. In addition, there exist some $t_{0}$ and $\delta>0$ such that

$$
\int_{0}^{t_{0}} \int_{0}^{t_{0}} r^{-\delta} s^{-\delta}\|\sigma(r)\|_{L_{2}^{0}(W, U)}\|\sigma(s)\|_{L_{2}^{0}(W, U)} d r d s<\infty .
$$

\section{Main results}

In this section, we will show the approximate controllability of the stochastic system (1.1). We need to establish the existence of the solution for the stochastic control system and to show that the corresponding linear part is approximate controllability.

Definition 3.1 The system (1.1) is called approximately controllable on $[0, T]$ if

$$
\overline{\mathfrak{R}(T)}=U
$$

with $\mathfrak{R}(t)=\left\{x(t)=x(t, u): u \in L^{2}([0, T], V)\right\}$.

Consider the corresponding linear fractional deterministic control system to (1.1)

$$
\left\{\begin{array}{l}
{ }^{\mathrm{c}} D_{t}^{\alpha}[L x(t)]=A x(t)+B u(t), \quad t \in[0, T] \\
x(0)=\phi(0)
\end{array}\right.
$$

and define the relevant operators

$$
\Gamma_{0}^{T}=\int_{0}^{T}(T-s)^{\alpha-1} \mathscr{S}_{L}(T-s) B B^{*} \mathscr{S}_{L}^{*}(T-s) d s
$$

and

$$
R\left(\alpha, \Gamma_{0}^{T}\right)=\left(\alpha I+\Gamma_{0}^{T}\right)^{-1}
$$

where $B^{*}$ and $\mathscr{S}_{L}^{*}(T-s)$ denote the adjoint operators of $B$ and $\mathscr{S}_{L}(T-s)$, respectively. It is clear that the operator $\Gamma_{0}^{T}$ is a linear bounded operator. The fact that the linear Sobolevtype fractional control system (3.1) is approximately controllable on $[0, T]$ is equivalent to the next hypothesis (see, for example, Mahmudov and Denker [22]): 
$\left(\mathbb{H}_{0}\right) \alpha R\left(\alpha, \Gamma_{0}^{T}\right) \rightarrow 0$ in the strong operator topology, as $\alpha \rightarrow 0^{+}$.

Lemma 3.1 (Guendouzi and Idrissi [23]) For any $z_{T} \in L^{2}(\Omega ; U)$, there exists $\widehat{\varphi}(t) \in$ $L^{2}\left(\Omega, L^{2}\left(0, T ; L_{2}^{0}\right)\right)$ such that

$$
z_{T}=E z_{T}+\int_{0}^{T} \widehat{\varphi}(s) d B^{H}(s)
$$

For any $\lambda>0$ and $z_{T} \in L^{2}(\Omega ; U)$, we now define the control function $u^{\lambda}$ as follows:

$$
\begin{aligned}
u^{\lambda}(t)= & B^{*} \mathscr{S}_{L}^{*}(T-t)\left(\lambda I+\Gamma_{0}^{T}\right)^{-1}\left[-\mathscr{T}_{L}(T)(L \phi(0))+\mathrm{E} z_{T}+\int_{0}^{T} \widehat{\varphi}(s) d B^{H}(s)\right] \\
& -B^{*} \mathscr{S}_{L}^{*}(T-t) \int_{0}^{t}\left(\lambda I+\Gamma_{s}^{T}\right)^{-1}(T-s)^{\alpha-1} \mathscr{S}_{L}(T-s) f\left(s, x_{s}\right) d s \\
& -B^{*} \mathscr{S}_{L}^{*}(T-t) \int_{0}^{t}\left(\lambda I+\Gamma_{s}^{T}\right)^{-1}(T-s)^{\alpha-1} \mathscr{S}_{L}(T-s) \sigma(s) d B^{H}(s) .
\end{aligned}
$$

Theorem 3.1 (Pachpatte [24]) Let $N$ be a convex subset of a normed linear space $X$ and let $0 \in N$. If $\Phi: N \rightarrow N$ is a completely continuous operator and

$$
\Pi(\Phi):=\{x \in N: x=\gamma \Phi x \text { for some } \gamma \in(0,1)\}
$$

then either $\Phi$ has a fixed point or $\Pi(\Phi)$ is bounded.

Define the space

$$
\mathscr{B}_{a}=\left\{x: x \in C((-\infty, T], U) \text { with } x_{0}=\phi \in \mathscr{B}_{h}\right\}
$$

and let $\|\cdot\|_{a}$ be a seminorm defined by

$$
\|x\|_{a}=\|\phi\|_{\mathscr{B}_{h}}+\sup _{s \in[0, T]}\left(\mathrm{E}\|x(s)\|^{2}\right)^{\frac{1}{2}}, \quad x \in \mathscr{B}_{a}
$$

where $C((-\infty, T], U)$ denotes the space of all continuous $U$-valued stochastic process $\{\xi(t), t \in(-\infty, T]\}$.

Lemma 3.2 (Li and Liu [25]) Assume that $x \in \mathscr{B}_{a}$, then, for all $t \in[0, T], x_{t} \in \mathscr{B}_{h}$. Moreover,

$$
l\left(\mathrm{E}\|x(t)\|^{2}\right)^{1 / 2} \leq l \sup _{s \in[0, t]}\left(\mathrm{E}\|x(s)\|^{2}\right)^{1 / 2}+\left\|x_{0}\right\|_{\mathscr{B}_{h}},
$$

where $l=\int_{-\infty}^{0} h(s) d s$ is given in Sect. 2 .

Theorem 3.2 Assume the conditions $\left(\mathbb{B}_{1}\right),\left(\mathbb{B}_{2}\right)$ hold, then, for each $\lambda>0$ there exists a mild solution of (1.1) on $(-\infty, T]$, provided that there is a constant $\mathscr{K}>0$ such that $\frac{\mathcal{K}_{1} \mathscr{K}}{\mathcal{K}_{2}}>1$, where

$$
\mathcal{K}_{1}=1-24 l^{2}\left(\frac{M \tilde{M}_{1} T^{\alpha}}{\alpha \Gamma(\alpha)}\right)^{2} N_{f}\left(1+4\left(\frac{M^{2} \widetilde{M}_{1}^{2} M_{B} T^{\alpha}}{\alpha \lambda \Gamma(\alpha)^{2}}\right)^{2}\right)>0,
$$




$$
\begin{aligned}
\mathcal{K}_{2}= & 4 l^{2}\left(M M_{1} \widetilde{M}_{1}\right)^{2} \mathrm{E}\left\|\phi_{0}\right\|^{2}\left[1+12\left(\frac{M^{2} \widetilde{M}_{1}^{2} M_{B}^{2} T^{\alpha}}{\alpha \lambda \Gamma(\alpha)^{2}}\right)^{2}\right]+4\|\phi\|_{\mathscr{B}_{h}}^{2} \\
& +12 l^{2}\left(\frac{M \widetilde{M}_{1}}{\Gamma(\alpha)}\right)^{2}\left[1+4\left(\frac{M^{2} \widetilde{M}_{1}^{2} M_{B}^{2} T^{\alpha}}{\alpha \lambda \Gamma(\alpha)^{2}}\right)^{2}\right] \times\left(\frac{2 k_{f} T^{2 \alpha}}{\alpha^{2}}+c H(2 H-1) N_{\sigma}\right) \\
& +96 l^{2}\left(\frac{M^{2} \widetilde{M}_{1}^{2} M_{B}^{2} T^{\alpha}}{\alpha \lambda \Gamma(\alpha)^{2}}\right)^{2}\left(\mathrm{E}\left\|z_{T}\right\|^{2}+c H(2 H-1) T^{2 H-1} \int_{0}^{T}\|\widehat{\varphi}(s)\|_{L_{2}^{0}(W, U)}^{2} d s\right)
\end{aligned}
$$

and $M_{B}=\|B\|$.

Proof Define the operator $\Phi: \mathscr{B}_{a} \rightarrow \mathscr{B}_{a}$ by

$$
\Phi x(t)= \begin{cases}\phi(t), & t \in(-\infty, 0], \\ \mathscr{T}_{L}(t)(L \phi(0))+\int_{0}^{t}(t-s)^{\alpha-1} \mathscr{S}_{L}(t-s)\left[f\left(s, x_{s}\right)+B u^{\lambda}(s)\right] d s & \\ \quad+\int_{0}^{t}(t-s)^{\alpha-1} \mathscr{S}_{L}(t-s) \sigma(s) d B^{H}(s), & t \in(0, T],\end{cases}
$$

for $x \in \mathscr{B}_{a}$.

We will show that $\Phi$ has a fixed point which is a mild solution for system (1.1). For $\phi \in \mathscr{B}_{h}$, define

$$
\widetilde{\phi}(t)= \begin{cases}\phi(t), & t \in(-\infty, 0], \\ \mathscr{T}_{L}(t)(L \phi(0)), & t \in(0, T] .\end{cases}
$$

Then $\widetilde{\phi}(t) \in \mathscr{B}_{a}$. Let $x(t)=\widetilde{\phi}(t)+y(t), t \in(-\infty, T]$. It is easy to check that $x(t)$ satisfies $(1.1)$ if and only if $y_{0}=0$ and

$$
\begin{aligned}
y(t)= & \int_{0}^{t}(t-s)^{\alpha-1} \mathscr{S}_{L}(t-s)\left[f\left(s, y_{s}+\widetilde{\phi}_{s}\right)+B u^{\lambda}(s)\right] d s \\
& +\int_{0}^{t}(t-s)^{\alpha-1} \mathscr{S}_{L}(t-s) \sigma(s) d B^{H}(s) .
\end{aligned}
$$

Denote $\mathscr{B}_{b}=\left\{y \in \mathscr{B}_{a}, y_{0}=0 \in \mathscr{B}_{h}\right\}$ and let $\|\cdot\|_{b}$ be the seminorm in $\mathscr{B}_{b}$, defined by

$$
\|y\|_{b}=\left\|y_{0}\right\|_{\mathscr{B}_{h}}+\sup _{s \in[0, T]}\left(\mathrm{E}\|y(s)\|^{2}\right)^{1 / 2}=\sup _{s \in[0, T]}\left(\mathrm{E}\|y(s)\|^{2}\right)^{1 / 2}
$$

For $r \geq 0$, we set $B_{r}=\left\{y \in \mathscr{B}_{b},\|y\|_{b}^{2} \leq r\right\}$. Then $B_{r}$ is a bounded closed convex set in $\mathscr{B}_{b}$ for each $r$. According to Lemma 3.2, we get

$$
\begin{aligned}
\left\|y_{t}+\widetilde{\phi}_{t}\right\|_{\mathscr{B}_{h}}^{2} & \leq 2\left(\left\|y_{t}\right\|_{\mathscr{B}_{h}}^{2}+\left\|\widetilde{\phi}_{t}\right\|_{\mathscr{B}_{h}}^{2}\right) \\
& \leq 4\left(l^{2} \sup _{s \in[0, t]} \mathrm{E}\|y(s)\|^{2}+\left\|y_{0}\right\|_{\mathscr{B}_{h}}^{2}+l^{2} \sup _{s \in[0, t]} \mathrm{E}\|\widetilde{\phi}(s)\|^{2}+\left\|\widetilde{\phi}_{0}\right\|_{\mathscr{B}_{h}}^{2}\right) \\
& \leq 4\left(l^{2} \sup _{s \in[0, t]} \mathrm{E}\|y(s)\|^{2}+l^{2} \sup _{s \in[0, t]} \mathrm{E}\left\|\mathscr{T}_{L}(t)(L \phi(0))\right\|^{2}+\|\phi\|_{\mathscr{B}_{h}}^{2}\right) \\
& \leq 4\left(l^{2} r+l^{2} M^{2} M_{1}^{2} \widetilde{M}_{1}^{2} \mathrm{E}\|\phi(0)\|^{2}+\|\phi\|_{\mathscr{B}_{h}}^{2}\right) \equiv r^{\prime}
\end{aligned}
$$


for $y \in B_{r}$. Define the mapping $\Psi: \mathscr{B}_{b} \rightarrow \mathscr{B}_{b}$ by

$$
\begin{aligned}
\Psi y(t)= & \int_{0}^{t}(t-s)^{\alpha-1} \mathscr{S}_{L}(t-s)\left[f\left(s, \widetilde{\phi}_{s}+y_{s}\right)+B u^{\lambda}(s)\right] d s \\
& +\int_{0}^{t}(t-s)^{\alpha-1} \mathscr{S}_{L}(t-s) \sigma(s) d B^{H}(s)
\end{aligned}
$$

for $t \in[0, T]$. It is evident that the operator $\Phi$ has a fixed point if and only if the operator $\Psi$ has a fixed point. Now, we turn to prove $\Psi$ has a fixed point.

Step I. We claim that $\Psi$ maps bounded sets into bounded sets of $\mathscr{B}_{b}$. This is equivalent to show that there exists a positive constant $\rho$ such that, for each $y \in B_{r}$, one has

$$
\|\Psi y(t)\|_{a}^{2} \leq \rho .
$$

By (3.2), we obtain

$$
\begin{aligned}
\mathrm{E}\left\|u^{\lambda}(s)\right\|^{2} \leq & \frac{4\left(M_{B} M \tilde{M}_{1}\right)^{2}}{\lambda^{2} \Gamma(\alpha)^{2}}\left(\mathrm{E}\left\|\mathscr{T}_{L}(T)(L \phi(0))\right\|^{2}+\mathrm{E}\left\|\mathrm{E} z_{T}+\int_{0}^{T} \widehat{\varphi}(s) d B^{H}(s)\right\|^{2}\right. \\
& +\mathrm{E}\left\|\int_{0}^{T}(T-s)^{\alpha-1} \mathscr{S}_{L}(T-s) f\left(s, y_{s}+\widetilde{\phi}_{s}\right) d s\right\|^{2} \\
& \left.+\mathrm{E}\left\|\int_{0}^{T}(T-s)^{\alpha-1} \mathscr{S}_{L}(T-s) \sigma(s) d B^{H}(s)\right\|^{2}\right) \\
\equiv & \frac{4\left(M_{B} M \tilde{M}_{1}\right)^{2}}{\lambda^{2} \Gamma(\alpha)^{2}} \sum_{i=1}^{4} I_{i} .
\end{aligned}
$$

By Lemma 2.2, it is easy to show that

$$
\begin{aligned}
I_{1}+I_{2} \leq & \left(M \widetilde{M}_{1} M_{1}\right)^{2} \mathrm{E}\|\phi(0)\|^{2}+2 \mathrm{E}\left\|z_{T}\right\|^{2} \\
& +2 c H(2 H-1) T^{2 H-1} \int_{0}^{T}\|\widehat{\varphi}(s)\|_{L_{2}^{0}(W, U)}^{2} d s .
\end{aligned}
$$

By using the Hölder inequality, the assumption (BB1), (3.3) and Lemma 2.3, we have

$$
\begin{aligned}
I_{3} & \leq \int_{0}^{T}(T-s)^{\alpha-1}\left\|\mathscr{S}_{L}(T-s)\right\| d s \int_{0}^{T}(T-s)^{\alpha-1}\left\|\mathscr{S}_{L}(T-s)\right\| \mathrm{E}\left\|f\left(s, y_{s}+\widetilde{\phi}_{s}\right)\right\|^{2} d s \\
& \leq 2\left(\frac{M \tilde{M}_{1}}{\Gamma(\alpha)}\right)^{2} \frac{T^{\alpha}}{\alpha} \int_{0}^{T}(T-s)^{\alpha-1}\left(N_{f}\left\|y_{s}+\widetilde{\phi}_{s}\right\|_{\mathscr{B}_{h}}^{2}+k_{f}\right) d s \\
& \leq 2\left(\frac{M \tilde{M}_{1} T^{\alpha}}{\alpha \Gamma(\alpha)}\right)^{2}\left(N_{f} r^{\prime}+k_{f}\right) \equiv \mu_{f} .
\end{aligned}
$$

For the last part $I_{4}$, when $\alpha>1-H$, we have

$$
\int_{0}^{T} \int_{0}^{T}(T-s)^{\alpha-1}(T-t)^{\alpha-1}|s-t|^{2 H-2} d s d t=\frac{B(\alpha, 2 H-1)}{2(\alpha+H-1)} T^{2(\alpha+H-1)}
$$


and

$$
N_{\sigma}:=\sup _{t \in[0, T]} \int_{0}^{t} \int_{0}^{t}(t-s)^{\alpha-1}(t-r)^{\alpha-1}|s-r|^{2 H-2}\|\sigma(s)\|_{L_{2}^{0}(W, U)}\|\sigma(r)\|_{L_{2}^{0}(W, U)} d s d r<\infty,
$$

where $B(\cdot, \cdot)$ denotes the Beta function, which imply that

$$
\begin{aligned}
I_{4}= & \mathrm{E}\left\|\sum_{n=1}^{\infty} \int_{0}^{T}(T-s)^{\alpha-1} \mathscr{S}_{L}(T-s) \sigma(s) Q^{\frac{1}{2}} e_{n} d \beta_{n}^{H}(s)\right\|^{2} \\
= & \sum_{n=1}^{\infty} \mathrm{E}\left\|\int_{0}^{T}(T-s)^{\alpha-1} \mathscr{S}_{L}(T-s) \sigma(s) Q^{\frac{1}{2}} e_{n} d \beta_{n}^{H}(s)\right\|^{2} \\
= & \sum_{n=1}^{\infty} H(2 H-1) \int_{0}^{T} \int_{0}^{T}\left\|(T-s)^{\alpha-1} \mathscr{S}_{L}(T-s) \sigma(s) Q^{\frac{1}{2}} e_{n}\right\| \\
& \times\left\|(T-t)^{\alpha-1} \mathscr{S}_{L}(T-t) \sigma(t) Q^{\frac{1}{2}} e_{n}\right\||s-t|^{2 H-2} d s d t \\
\leq & c H(2 H-1)\left(\frac{M \tilde{M}_{1}}{\Gamma(\alpha)}\right)^{2} \int_{0}^{T} \int_{0}^{T}(T-s)^{\alpha-1}(T-t)^{\alpha-1}|s-t|^{2 H-2} \\
& \times\|\sigma(s)\|_{L_{2}^{0}(W, U)}\|\sigma(t)\|_{L_{2}^{0}(W, U)} d s d t .
\end{aligned}
$$

Consequently, we get

$$
\begin{aligned}
\mathrm{E}\left\|u^{\lambda}(s)\right\|^{2} \leq & \frac{4\left(M_{B} M \tilde{M}_{1}\right)^{2}}{\lambda^{2} \Gamma(\alpha)^{2}}\left\{\left(M \widetilde{M}_{1} M_{1}\right)^{2} \mathrm{E}\|\phi(0)\|^{2}+2 \mathrm{E}\left\|z_{T}\right\|^{2}\right. \\
& +2 c H(2 H-1) T^{2 H-1} \int_{0}^{T}\|\widehat{\varphi}(s)\|_{L_{2}^{0}(W, U)}^{2} d s+\mu_{f} \\
& \left.+c H(2 H-1)\left(\frac{M \widetilde{M}_{1}}{\Gamma(\alpha)}\right)^{2} N_{\sigma}\right\} \\
\equiv & \frac{4\left(M_{B} M \widetilde{M}_{1}\right)^{2}}{\lambda^{2} \Gamma(\alpha)^{2}} N_{u} .
\end{aligned}
$$

It follows that

$$
\begin{aligned}
\mathrm{E}\|\Psi y(t)\|^{2} \leq & 3 \mathrm{E}\left\|\int_{0}^{t}(t-s)^{\alpha-1} \mathscr{S}_{L}(t-s) f\left(s, y_{s}+\widetilde{\phi}_{s}\right) d s\right\|^{2} \\
& +3 \mathrm{E}\left\|\int_{0}^{t}(t-s)^{\alpha-1} \mathscr{S}_{L}(t-s) B u^{\lambda}(s) d s\right\|^{2} \\
& +3 \mathrm{E}\left\|\int_{0}^{t}(t-s)^{\alpha-1} \mathscr{S}_{L}(t-s) \sigma(s) d B^{H}(s)\right\|^{2} \\
\leq & 3\left(\frac{M \widetilde{M}_{1}}{\Gamma(\alpha)}\right)^{2}\left[\frac{2 T^{2 \alpha}}{\alpha^{2}}\left(N_{f} r^{\prime}+k_{f}\right)\right. \\
& \left.+\left(\frac{M_{B} T^{\alpha}}{\alpha}\right)^{2} \frac{4\left(M_{B} M \widetilde{M}_{1}\right)^{2}}{\lambda^{2} \Gamma(\alpha)^{2}} N_{u}+c H(2 H-1) N_{\sigma}\right] \equiv \rho<\infty .
\end{aligned}
$$

This shows that the first statement holds, i.e., for each $y \in B_{r},\|\Psi y(t)\|_{a}^{2} \leq \rho$. 
Step II. We claim that $\Psi$ maps bounded sets into equicontinuous sets of $\mathscr{B}_{b}$. Similar to the calculation in Step I, for $0 \leq t_{1}<t_{2} \leq T$, we have

$$
\begin{aligned}
\mathrm{E} \| & \Psi\left(t_{2}\right)-\Psi y\left(t_{1}\right) \|^{2} \\
\leq & 3 \mathrm{E} \| \int_{0}^{t_{2}}\left(t_{2}-s\right)^{\alpha-1} \mathscr{S}_{L}\left(t_{2}-s\right) f\left(s, y_{s}+\widetilde{\phi}_{s}\right) d s \\
& -\int_{0}^{t_{1}}\left(t_{1}-s\right)^{\alpha-1} \mathscr{S}_{L}\left(t_{1}-s\right) f\left(s, y_{s}+\widetilde{\phi}_{s}\right) d s \|^{2} \\
& +3 \mathrm{E}\left\|\int_{0}^{t_{2}}\left(t_{2}-s\right)^{\alpha-1} \mathscr{S}_{L}\left(t_{2}-s\right) B u^{\lambda}(s) d s-\int_{0}^{t_{1}}\left(t_{1}-s\right)^{\alpha-1} \mathscr{S}_{L}\left(t_{1}-s\right) B u^{\lambda}(s) d s\right\|^{2} \\
& +3 \mathrm{E} \| \int_{0}^{t_{2}}\left(t_{2}-s\right)^{\alpha-1} \mathscr{S}_{L}\left(t_{2}-s\right) \sigma(s) d B^{H}(s) \\
& -\int_{0}^{t_{1}}\left(t_{1}-s\right)^{\alpha-1} \mathscr{S}_{L}\left(t_{1}-s\right) \sigma(s) d B^{H}(s) \|^{2} \\
\equiv & \sum_{j=1}^{3} J_{j} .
\end{aligned}
$$

We estimate $J_{1}, J_{2}, J_{3}$. Let $0<\varepsilon<t<T$ and $\delta>0$ such that

$$
\left\|\mathscr{S}_{L}\left(s_{2}\right)-\mathscr{S}_{L}\left(s_{1}\right)\right\|<\varepsilon
$$

for every $s_{1}, s_{2} \in[0, T]$ with $\left|s_{1}-s_{2}\right|<\delta$. Then, for $J_{1}$ we have

$$
\begin{aligned}
J_{1} \leq & 3 \mathrm{E}\left\|\int_{0}^{t_{1}}\left[\left(t_{2}-s\right)^{\alpha-1}-\left(t_{1}-s\right)^{\alpha-1}\right] \mathscr{S}_{L}\left(t_{2}-s\right) f\left(s, y_{s}+\widetilde{\phi}_{s}\right) d s\right\|^{2} \\
& +3 \mathrm{E}\left\|\int_{0}^{t_{1}}\left(t_{1}-s\right)^{\alpha-1}\left[\mathscr{S}_{L}\left(t_{2}-s\right)-\mathscr{S}_{L}\left(t_{1}-s\right)\right] f\left(s, y_{s}+\widetilde{\phi}_{s}\right) d s\right\|^{2} \\
& +3 \mathrm{E}\left\|\int_{t_{1}}^{t_{2}}\left(t_{2}-s\right)^{\alpha-1} \mathscr{S}_{L}\left(t_{2}-s\right) f\left(s, y_{s}+\widetilde{\phi}_{s}\right) d s\right\|^{2} \\
\leq & 3\left(\frac{M \tilde{M}_{1}}{\Gamma(\alpha)}\right)^{2} \int_{0}^{t_{1}}\left[\left(t_{1}-s\right)^{\alpha-1}-\left(t_{2}-s\right)^{\alpha-1}\right] d s \\
& \times \int_{0}^{t_{1}}\left[\left(t_{1}-s\right)^{\alpha-1}-\left(t_{2}-s\right)^{\alpha-1}\right] \mathrm{E}\left\|f\left(s, y_{s}+\widetilde{\phi}_{s}\right)\right\|^{2} d s \\
& +\frac{3 t_{1}^{\alpha} \varepsilon^{2}}{\alpha} \int_{0}^{t_{1}}\left(t_{1}-s\right)^{\alpha-1} \mathrm{E}\left\|f\left(s, y_{s}+\widetilde{\phi}_{s}\right)\right\|^{2} d s \\
& +\frac{3\left(t_{2}-t_{1}\right)^{\alpha}}{\alpha}\left(\frac{M \tilde{M}_{1}}{\Gamma(\alpha)}\right)^{2} \int_{t_{1}}^{t_{2}}\left(t_{2}-s\right)^{\alpha-1} \mathrm{E}\left\|f\left(s, y_{s}+\widetilde{\phi}_{s}\right)\right\|^{2} d s \\
\leq & 3\left(\frac{M \tilde{M}_{1}}{\Gamma(\alpha)}\right)^{2}\left(N_{f} r^{\prime}+k_{f}\right)\left(\int_{0}^{t_{1}}\left[\left(t_{1}-s\right)^{\alpha-1}-\left(t_{2}-s\right)^{\alpha-1}\right] d s\right)^{2} \\
& +\frac{3 t_{1}^{2 \alpha} \varepsilon^{2}}{\alpha^{2}}\left(N_{f} r^{\prime}+k_{f}\right)+\frac{3\left(t_{2}-t_{1}\right)^{2 \alpha}}{\alpha^{2}}\left(\frac{M \tilde{M}_{1}}{\Gamma(\alpha)}\right)^{2}\left(N_{f} r^{\prime}+k_{f}\right) .
\end{aligned}
$$


Analogously, for $J_{2}$ we also have

$$
\begin{aligned}
J_{2} \leq & 3 \mathrm{E}\left\|\int_{0}^{t_{1}}\left[\left(t_{2}-s\right)^{\alpha-1}-\left(t_{1}-s\right)^{\alpha-1}\right] \mathscr{S}_{L}\left(t_{2}-s\right) B u^{\lambda}(s) d s\right\|^{2} \\
& +3 \mathrm{E}\left\|\int_{0}^{t_{1}}\left(t_{1}-s\right)^{\alpha-1}\left[\mathscr{S}_{L}\left(t_{2}-s\right)-\mathscr{S}_{L}\left(t_{1}-s\right)\right] B u^{\lambda}(s) d s\right\|^{2} \\
& +3 \mathrm{E}\left\|\int_{t_{1}}^{t_{2}}\left(t_{2}-s\right)^{\alpha-1} \mathscr{S}_{L}\left(t_{2}-s\right) B u^{\lambda}(s) d s\right\|^{2} \\
\leq & 3\left(\frac{M \tilde{M}_{1} M_{B}}{\Gamma(\alpha)}\right)^{2} \int_{0}^{t_{1}}\left[\left(t_{1}-s\right)^{\alpha-1}-\left(t_{2}-s\right)^{\alpha-1}\right] d s \\
& \times \int_{0}^{t_{1}}\left[\left(t_{1}-s\right)^{\alpha-1}-\left(t_{2}-s\right)^{\alpha-1}\right] \mathrm{E}\left\|u^{\lambda}(s)\right\|^{2} d s \\
& +\frac{3 M_{B}^{2} t_{1}^{\alpha} \varepsilon^{2}}{\alpha} \int_{0}^{t_{1}}\left(t_{1}-s\right)^{\alpha-1} \mathrm{E}\left\|u^{\lambda}(s)\right\|^{2} d s \\
& +\frac{3\left(t_{2}-t_{1}\right)^{\alpha}}{\alpha}\left(\frac{M \tilde{M}_{1} M{ }_{B}}{\Gamma(\alpha)}\right)^{2} \int_{t_{1}}^{t_{2}}\left(t_{2}-s\right)^{\alpha-1} \mathrm{E}\left\|u^{\lambda}(s)\right\|^{2} d s \\
\leq & 3\left(\frac{M \tilde{M}_{1} M_{B}}{\Gamma(\alpha)}\right)^{2} \frac{4\left(M \tilde{M}_{1}\right)^{2}}{\lambda^{2} \Gamma(\alpha)^{2}} N_{u}\left(\int_{0}^{t_{1}}\left[\left(t_{1}-s\right)^{\alpha-1}-\left(t_{2}-s\right)^{\alpha-1}\right] d s\right)^{2} \\
& \frac{3 t_{1}^{2 \alpha} M_{B}^{2} \varepsilon^{2}}{\alpha^{2}} \frac{4\left(M_{B} M \tilde{M}_{1}\right)^{2}}{\lambda^{2} \Gamma(\alpha)^{2}} N_{u}+\frac{3\left(t_{2}-t_{1}\right)^{2 \alpha}}{\alpha^{2}}\left(\frac{M \tilde{M}_{1} M_{B}}{\Gamma(\alpha)}\right)^{2} \frac{4\left(M_{B} M \tilde{M}_{1}\right)^{2}}{\lambda^{2} \Gamma(\alpha)^{2}} N_{u} .
\end{aligned}
$$

As $\alpha>1-H$, for $J_{3}$

$$
\begin{aligned}
J_{3} \leq & 3 \mathrm{E}\left\|\int_{0}^{t_{1}}\left[\left(t_{2}-s\right)^{\alpha-1}-\left(t_{1}-s\right)^{\alpha-1}\right] \mathscr{S}_{L}\left(t_{2}-s\right) \sigma(s) d B^{H}(s)\right\|^{2} \\
& +3 \mathrm{E}\left\|\int_{0}^{t_{1}}\left(t_{1}-s\right)^{\alpha-1}\left[\mathscr{S}_{L}\left(t_{2}-s\right)-\mathscr{S}_{L}\left(t_{1}-s\right)\right] \sigma(s) d B^{H}(s)\right\|^{2} \\
& +3 \mathrm{E}\left\|\int_{t_{1}}^{t_{2}}\left(t_{2}-s\right)^{\alpha-1} \mathscr{S}_{L}\left(t_{2}-s\right) \sigma(s) d B^{H}(s)\right\|^{2} \\
\leq & 3 c H(2 H-1)\left(\frac{M \tilde{M}_{1}}{\Gamma(\alpha)}\right)^{2} \int_{0}^{t_{1}} \int_{0}^{t_{1}}\left[\left(t_{1}-s\right)^{\alpha-1}-\left(t_{2}-s\right)^{\alpha-1}\right] \\
& \times\left[\left(t_{1}-t\right)^{\alpha-1}-\left(t_{2}-t\right)^{\alpha-1}\right] \\
& \times\|\sigma(s)\|_{L_{2}^{0}(W, U)}\|\sigma(t)\|_{L_{2}^{0}(W, U)}|s-t|^{2 H-2} d s d t \\
& +3 c H(2 H-1) \varepsilon^{2} N_{\sigma} \\
& +3 c H(2 H-1)\left(\frac{M \tilde{M}_{1}}{\Gamma(\alpha)}\right)^{2} \int_{t_{1}}^{t_{2}} \int_{t_{1}}^{t_{2}}\left(t_{2}-s\right)^{\alpha-1}\left(t_{2}-t\right)^{\alpha-1}|s-t|^{2 H-2} \\
& \times\|\sigma(s)\|_{L_{2}^{0}(W, U)}\|\sigma(t)\|_{L_{2}^{0}(W, U)} d s d t .
\end{aligned}
$$

Thus, we see that $J_{j}(j=1,2,3)$ tends to zero, as $t_{1} \rightarrow t_{2}$. It follows from the Arzela-Ascoli theorem that $\Psi$ is completely continuous.

Step III. We show that there exists an open set $\Pi \subseteq \mathscr{B}_{b}$ with $y \neq \gamma \Psi y$ for $\gamma \in(0,1)$ and $y \in \partial \Pi$. 
If $y=\gamma \Psi y \in \mathscr{B}_{b}$ for some $\gamma \in(0,1)$. We then have

$$
\begin{aligned}
y(t)= & \gamma\left\{\int_{0}^{t}(t-s)^{\alpha-1} \mathscr{S}_{L}(t-s)\left[f\left(s, y_{s}+\widetilde{\phi}_{s}\right)+B u^{\lambda}(s)\right] d s\right. \\
& \left.+\int_{0}^{t}(t-s)^{\alpha-1} \mathscr{S}_{L}(t-s) \sigma(s) d B^{H}(s)\right\}
\end{aligned}
$$

for every $t \in[0, T]$. It follows that

$$
\begin{aligned}
\mathrm{E}\|y(t)\|^{2} \leq & 3 \mathrm{E}\left\|\int_{0}^{t}(t-s)^{\alpha-1} \mathscr{S}_{L}(t-s) f\left(s, y_{s}+\widetilde{\phi}_{s}\right) d s\right\|^{2} \\
& +3 \mathrm{E}\left\|\int_{0}^{t}(t-s)^{\alpha-1} \mathscr{S}_{L}(t-s) B u^{\lambda}(s) d s\right\|^{2} \\
& +3 \mathrm{E}\left\|\int_{0}^{t}(t-s)^{\alpha-1} \mathscr{S}_{L}(t-s) \sigma(s) d B^{H}(s)\right\|^{2} \\
\leq & 6\left(\frac{M \widetilde{M}_{1} T^{\alpha}}{\alpha \Gamma(\alpha)}\right)^{2} N_{f}\left\|y_{t}+\widetilde{\phi}_{t}\right\|_{\mathscr{B}_{h}}^{2}+6\left(\frac{M \widetilde{M}_{1} T^{\alpha}}{\alpha \Gamma(\alpha)}\right)^{2} k_{f} \\
& +3\left(\frac{M \widetilde{M}_{1} M_{B} T^{\alpha}}{\alpha \Gamma(\alpha)}\right)^{2} \mathrm{E}\left\|u^{\lambda}(t)\right\|^{2}+3\left(\frac{M \tilde{M}_{1}}{\Gamma(\alpha)}\right)^{2} c H(2 H-1) N_{\sigma},
\end{aligned}
$$

which implies that

$$
\begin{aligned}
\left\|y_{t}+\widetilde{\phi}_{t}\right\|_{\mathscr{B}_{h}}^{2} & \leq 2\left(\left\|y_{t}\right\|_{\mathscr{B}_{h}}^{2}+\left\|\widetilde{\phi}_{t}\right\|_{\mathscr{B}_{h}}^{2}\right) \\
& \leq 4\left(l^{2} \sup _{s \in[0, t]} \mathrm{E}\|y(s)\|^{2}+l^{2} M^{2} M_{1}^{2} \widetilde{M}_{1}^{2} \mathrm{E}\|\phi(0)\|^{2}+\|\phi\|_{\mathscr{B}_{h}}^{2}\right) .
\end{aligned}
$$

Denote the right side of the above inequality by $\psi(t)$. There exists $t^{*} \in[0, t]$ such that

$$
\psi(t)=4\left(l^{2} \mathrm{E}\left\|y\left(t^{*}\right)\right\|^{2}+l^{2} M^{2} M_{1}^{2} \widetilde{M}_{1}^{2} \mathrm{E}\|\phi(0)\|^{2}+\|\phi\|_{\mathscr{B}_{h}}^{2}\right) .
$$

Then, for $t \in[0, T]$, we have

$$
\begin{aligned}
\psi(t) \leq & 4\left\{l ^ { 2 } \left[6\left(\frac{M \tilde{M}_{1} T^{\alpha}}{\alpha \Gamma(\alpha)}\right)^{2} N_{f} \psi(t)+6\left(\frac{M \tilde{M}_{1} T^{\alpha}}{\alpha \Gamma(\alpha)}\right)^{2} k_{f}\right.\right. \\
& +12\left(\frac{M^{2} \widetilde{M}_{1}^{2} M_{B}^{2} T^{\alpha}}{\lambda \alpha \Gamma(\alpha)^{2}}\right)^{2} \times\left(\left(M \widetilde{M}_{1} M_{1}\right)^{2} \mathrm{E}\|\phi(0)\|^{2}+2 \mathrm{E}\left\|z_{T}\right\|^{2}\right. \\
& +2 c H(2 H-1) T^{2 H-1} \int_{0}^{T}\|\widehat{\varphi}(s)\|_{L_{2}^{0}(W, U)}^{2} d s+2\left(\frac{M \tilde{M}_{1} T^{\alpha}}{\alpha \Gamma(\alpha)}\right)^{2} N_{f} \psi(t) \\
& \left.+2\left(\frac{M \tilde{M}_{1} T^{\alpha}}{\alpha \Gamma(\alpha)}\right)^{2} k_{f}+c H(2 H-1)\left(\frac{M \tilde{M}_{1}}{\Gamma(\alpha)}\right)^{2} N_{\sigma}\right) \\
& \left.\left.+3\left(\frac{M \widetilde{M}_{1}}{\Gamma(\alpha)}\right)^{2} c H(2 H-1) N_{\sigma}\right]+l^{2} M^{2} M_{1}^{2} \widetilde{M}_{1}^{2} \mathrm{E}\|\phi(0)\|^{2}+\|\phi\|_{\mathscr{B}_{h}}^{2}\right\}
\end{aligned}
$$


Taking the norm on both sides above, it follows that

$$
\begin{aligned}
\|\psi(t)\|_{a}^{2} \leq & 4 l^{2}\left[6\left(\frac{M \tilde{M}_{1} T^{\alpha}}{\alpha \Gamma(\alpha)}\right)^{2} N_{f}\|\psi(t)\|_{a}^{2}+6\left(\frac{M \tilde{M}_{1} T^{\alpha}}{\alpha \Gamma(\alpha)}\right)^{2} k_{f}\right. \\
& +12\left(\frac{M^{2} \tilde{M}_{1}^{2} M_{B}^{2} T^{\alpha}}{\lambda \alpha \Gamma(\alpha)^{2}}\right)^{2} \times\left(\left(M \tilde{M}_{1} M_{1}\right)^{2} \mathrm{E}\|\phi(0)\|^{2}+2 \mathrm{E}\left\|z_{T}\right\|^{2}\right. \\
& +2 c H(2 H-1) T^{2 H-1} \int_{0}^{T}\|\widehat{\varphi}(s)\|_{L_{2}^{0}(W, U)}^{2} d s+2\left(\frac{M \tilde{M}_{1} T^{\alpha}}{\alpha \Gamma(\alpha)}\right)^{2} N_{f}\|\psi(t)\|_{a}^{2} \\
& \left.+2\left(\frac{M \tilde{M}_{1} T^{\alpha}}{\alpha \Gamma(\alpha)}\right)^{2} k_{f}+c H(2 H-1)\left(\frac{M \tilde{M}_{1}}{\Gamma(\alpha)}\right)^{2} N_{\sigma}\right) \\
& \left.\left.+3\left(\frac{M \tilde{M}_{1}}{\Gamma(\alpha)}\right)^{2} c H(2 H-1) N_{\sigma}\right]+l^{2} M^{2} M_{1}^{2} \tilde{M}_{1}^{2} \mathrm{E}\|\phi(0)\|^{2}+\|\phi\|_{\mathscr{B}_{h}}^{2}\right\},
\end{aligned}
$$

which implies that $\frac{\mathcal{K}_{1}\|\psi(t)\|_{a}^{2}}{\mathcal{K}_{2}} \leq 1$, where

$$
\mathcal{K}_{1}=1-24 l^{2}\left(\frac{M \tilde{M}_{1} T^{\alpha}}{\alpha \Gamma(\alpha)}\right)^{2} N_{f}\left(1+4\left(\frac{M^{2} \tilde{M}_{1}^{2} M_{B} T^{\alpha}}{\alpha \lambda \Gamma(\alpha)^{2}}\right)^{2}\right)
$$

and

$$
\begin{aligned}
\mathcal{K}_{2}= & 4 l^{2}\left(M M_{1} \tilde{M}_{1}\right)^{2} \mathrm{E}\left\|\phi_{0}\right\|^{2}\left[1+12\left(\frac{M^{2} \tilde{M}_{1}^{2} M_{B}^{2} T^{\alpha}}{\alpha \lambda \Gamma(\alpha)^{2}}\right)^{2}\right]+4\|\phi\|_{\mathscr{B}_{h}}^{2} \\
& +12 l^{2}\left(\frac{M \tilde{M}_{1}}{\Gamma(\alpha)}\right)^{2}\left[1+4\left(\frac{M^{2} \tilde{M}_{1}^{2} M_{B}^{2} T^{\alpha}}{\alpha \lambda \Gamma(\alpha)^{2}}\right)^{2}\right] \times\left(\frac{2 k_{f} T^{2 \alpha}}{\alpha^{2}}+c H(2 H-1) N_{\sigma}\right) \\
& +96 l^{2}\left(\frac{M^{2} \tilde{M}_{1}^{2} M_{B}^{2} T^{\alpha}}{\alpha \lambda \Gamma(\alpha)^{2}}\right)^{2}\left(\mathrm{E}\left\|z_{T}\right\|^{2}+c H(2 H-1) T^{2 H-1} \int_{0}^{T}\|\widehat{\varphi}(s)\|_{L_{2}^{0}(W, U)}^{2} d s\right) .
\end{aligned}
$$

From the assumption, $\|\psi\|_{a}^{2} \neq \mathscr{K}$. Set $\Pi=\left\{y \in \mathscr{B}_{b},\|y\|_{a}^{2}<\mathscr{K}+1\right\}$. Then there is no $y \in \partial \Pi$ such that $y=\gamma \Psi y$ for some $\gamma \in(0,1)$. By Theorem 3.1, we find that $\Psi$ has a fixed point. Hence $\Phi$ has a fixed point which is a solution to the system (1.1).

Theorem 3.3 Assume that the conditions of Theorem 3.2 and $\left(\mathbb{H}_{0}\right)$ hold. In addition, the functions $f$ is uniformly bounded on its domain. Then the fractional control system (1.1) is approximately controllable on $[0, T]$.

Proof Let $x^{\lambda}$ be a fixed point of the operator $\Phi^{\lambda}$. Using the stochastic Fubini theorem, we can get

$$
\begin{aligned}
x^{\lambda}(T)= & z_{T}-\lambda\left(\lambda I+\Gamma_{0}^{T}\right)^{-1}\left[-\mathscr{T}_{L}(T)(L \phi(0))+\mathrm{E} z_{T}+\int_{0}^{T} \widehat{\varphi}(s) d B^{H}(s)\right] \\
& +\lambda \int_{0}^{T}\left(\lambda I+\Gamma_{s}^{T}\right)^{-1}(T-s)^{\alpha-1} \mathscr{S}_{L}(T-s) f\left(s, x_{s}^{\lambda}\right) d s \\
& +\lambda \int_{0}^{T}\left(\lambda I+\Gamma_{s}^{T}\right)^{-1}(T-s)^{\alpha-1} \mathscr{S}_{L}(T-s) \sigma(s) d B^{H}(s) .
\end{aligned}
$$

It follows from the property of $f$ that there exists $C>0$ such that $\left\|f\left(s, x_{s}^{\lambda}\right)\right\|^{2} \leq C$. Then there is a subsequence denoted by $\left\{f\left(s, x_{s}^{\lambda}\right)\right\}$ weakly converging to $f(s)$. Thus, from the 
above equation, we obtain

$$
\begin{aligned}
& \mathrm{E}\left\|x^{\lambda}(T)-z_{T}\right\|^{2} \\
& \leq 5\left\|\lambda\left(\lambda I+\Gamma_{0}^{T}\right)^{-1}\left[\mathrm{E} z_{T}-\mathscr{T}_{L}(T)(L \phi(0))\right]\right\|^{2} \\
&+5 c H(2 H-1) T^{2 H-1} \mathrm{E} \int_{0}^{T}\left\|\lambda\left(\lambda I+\Gamma_{0}^{T}\right)^{-1} \widehat{\varphi}(s)\right\|_{L_{2}^{0}(W, U)}^{2} d s \\
&+5 \mathrm{E}\left(\int_{0}^{T}(T-s)^{\alpha-1}\left\|\lambda\left(\lambda I+\Gamma_{s}^{T}\right)^{-1} \mathscr{S}_{L}(T-s)\left[f\left(s, x_{s}^{\lambda}\right)-f(s)\right]\right\| d s\right)^{2} \\
&+5 \mathrm{E}\left(\int_{0}^{T}(T-s)^{\alpha-1}\left\|\lambda\left(\lambda I+\Gamma_{s}^{T}\right)^{-1} \mathscr{S}_{L}(T-s) f(s)\right\| d s\right)^{2} \\
&+5 c H(2 H-1) \int_{0}^{T} \int_{0}^{T}(T-s)^{\alpha-1}(T-t)^{\alpha-1}|s-t|^{2 H-2} \\
& \times\left\|\lambda\left(\lambda I+\Gamma_{s}^{T}\right)^{-1} \mathscr{S}_{L}(T-s) \sigma(s)\right\|_{L_{2}^{0}(W, U)}^{2} \\
& \times\left\|\lambda\left(\lambda I+\Gamma_{t}^{T}\right)^{-1} \mathscr{S}_{L}(T-t) \sigma(t)\right\|_{L_{2}^{0}(W, U)}^{2} d s d t .
\end{aligned}
$$

On the other hand, by assumption $\left(\mathbb{H}_{0}\right)$ for all $0 \leq s \leq T$, the operator $\lambda\left(\lambda I+\Gamma_{s}^{T}\right)^{-1} \rightarrow 0$ strongly as $\lambda \rightarrow 0+$, and, moreover, $\left\|\lambda\left(\lambda I+\Gamma_{s}^{T}\right)^{-1}\right\|<1$. Thus, by the Lebesgue dominated convergence theorem and the compactness of $\mathscr{S}_{L}(t)$, we can get $\mathrm{E}\left\|x^{\lambda}(T)-z_{T}\right\|^{2} \rightarrow 0$ as $\lambda \rightarrow 0+$. This gives the approximate controllability of (1.1).

\section{Example}

In this section, we will show an example to apply our results above. Consider the Sobolevtype fractional stochastic functional equation with infinite delay of the following form:

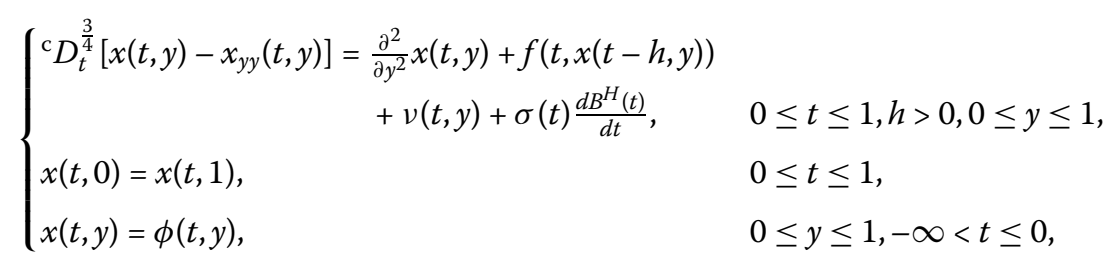

$B^{H}(t)$ is a cylindrical fractional Brownian motion with Hurst index $H \in\left(\frac{1}{2}, 1\right)$.

Take $U=L^{2}([0,1])$. Define the operators $A: D(A) \subset U \rightarrow U$ and $L: D(L) \subset U \rightarrow U$ by $A x=x^{\prime \prime}$ and $L x=x-x^{\prime \prime}$, where $D(A)$ and $D(L)$ are given by

$\left\{x \in U, x\right.$ and $x^{\prime}$ are absolutely continuous, $\left.x^{\prime \prime} \in U, x(0)=x(1)=0\right\}$.

Furthermore, $A$ and $L$ can be written as

$$
\begin{aligned}
& A x=\sum_{n=1}^{\infty} n^{2}\left\langle x, x_{n}\right\rangle x_{n}, \quad x \in D(A), \\
& L x=\sum_{n=1}^{\infty}\left(1+n^{2}\right)\left\langle x, x_{n}\right\rangle x_{n}, \quad x \in D(L),
\end{aligned}
$$


where $x_{n}(y)=\sqrt{\frac{2}{\pi}} \sin (n y), n=1,2, \ldots$, is the orthogonal set of eigenvectors of $A$. Also, for $x \in U$,

$$
\begin{aligned}
& L^{-1} x=\sum_{n=1}^{\infty} \frac{1}{1+n^{2}}\left\langle x, x_{n}\right\rangle x_{n}, \quad A L^{-1} x=\sum_{n=1}^{\infty} \frac{n^{2}}{1+n^{2}}\left\langle x, x_{n}\right\rangle x_{n}, \\
& S(t) x=\sum_{n=1}^{\infty} e^{\frac{-n^{2}}{1+n^{2}} t}\left\langle x, x_{n}\right\rangle x_{n}, \\
& \mathscr{S}_{L}(t)(x)=\frac{3}{4} \sum_{n=1}^{\infty} \frac{1}{1+n^{2}} \int_{0}^{\infty} \theta \xi_{\frac{3}{4}}(\theta) e^{\frac{-n^{2}}{1+n^{2}} t^{\frac{3}{4}} \theta} d \theta\left\langle x, x_{n}\right\rangle x_{n} .
\end{aligned}
$$

Clearly, $L^{-1}$ is compact, bounded with $\left\|L^{-1}\right\| \leq 1$ and $A L^{-1}$ generates a strongly continuous semigroup $S(t)$ on $U$ with $\|S(t)\| \leq 1$.

Let $h(s)=e^{2 s}, s<0$, then $l=\int_{-\infty}^{0} h(s) d s=\frac{1}{2}$. Let $\mathscr{B}_{h}$ be a phase space endowed with the norm

$$
\|\phi\|_{\mathscr{B}_{h}}=\int_{-\infty}^{0} h(s) \sup _{s \leq \theta \leq 0}\left(\mathrm{E}\|\phi(\theta)\|^{2}\right)^{\frac{1}{2}} d s .
$$

Then $\left(\mathscr{B}_{h},\|\cdot\|_{\mathscr{B}_{h}}\right)$ is a Banach space. Define an infinite-dimensional space $V$ by $V=$ $\left\{u \mid u=\sum_{n=2}^{\infty} u_{n} x_{n}\right.$, with $\left.\sum_{n=2}^{\infty} u_{n}^{2}<\infty\right\}$. The norm in $V$ is defined by $\|u\|_{V}=\left(\sum_{n=2}^{\infty} u_{n}^{2}\right)^{\frac{1}{2}}$. Then define a continuous linear mapping $B$ from $V$ into $U$ as $B u=2 u_{2} x_{1}+\sum_{n=2}^{\infty} u_{n} x_{n}$ for $u=\sum_{n=2}^{\infty} u_{n} x_{n} \in V$. We assume the operator $B: V \rightarrow U$ is a bounded linear operator by $B u(t)(y)=v(t, y)$. In addition, the linear part corresponding to (4.1) is approximately controllable. Then the system (4.1) can be written in the abstract form of (1.1). Imposing all the conditions of corresponding coefficients of Theorem 3.3, we can conclude that the fractional control system (4.1) is approximately controllable on $[0,1]$.

\section{Acknowledgements}

The Project-sponsored by National Natural Science Foundation of China (11571071).

\section{Competing interests}

The authors declare that they have no competing interests.

\section{Authors' contributions}

$\mathrm{JH}$ and LY carried out the mathematical studies, participated in the sequence alignment, drafted the manuscript and participated in the design of the study and performed proof of results. All authors read and approved the final manuscript.

\section{Author details}

${ }^{1}$ College of Information Science and Technology, Donghua University, Shanghai, P.R. China. ${ }^{2}$ Department of Mathematics, College of Science, Donghua University, Shanghai, P.R. China.

\section{Publisher's Note}

Springer Nature remains neutral with regard to jurisdictional claims in published maps and institutional affiliations.

Received: 2 February 2018 Accepted: 16 March 2018 Published online: 24 March 2018

\section{References}

1. Agarwal, R.P., Baghli, S., Benchohra, M.: Controllability for semilinear functional and neutral functional evolution equations with infinite delay in Fréchet spaces. Appl. Math. Optim. 60, 253-274 (2009)

2. Benchohra, M., Ouahab, A.: Controllability results for functional semilinear differential inclusions in Fréchet spaces. Nonlinear Anal. 61, 405-423 (2005)

3. Dauer, J.P., Mahmudov, N.ı: Controllability of stochastic semilinear functional differential equations in Hilbert spaces. J. Math. Anal. Appl. 290, 373-394 (2004) 
4. Górniewicz, L., Ntouyas, S.K., O'Regan, D.: Existence and controllability results for first- and second-order functional semilinear differential inclusions with nonlocal conditions. Numer. Funct. Anal. Optim. 28, 53-82 (2007)

5. Mahmudov, N.I.: Approximate controllability of semilinear deterministic and stochastic evolution equations in abstract spaces. SIAM J. Control Optim. 42, 1604-1622 (2003)

6. Mahmudov, N.I.: Approximate controllability of evolution systems with nonlocal conditions. Nonlinear Anal. 68 536-546 (2008)

7. Sakthivel, R., Ganesh, R., Suganya, S.: Approximate controllability of fractional neutral stochastic system with infinite delay. Rep. Math. Phys. 70, 291-311 (2012)

8. Sakthivel, R., Ren, Y., Mahmudov, N.I.: On the approximate controllability of semilinear fractional differential systems. Comput. Math. Appl. 62, 1451-1459 (2011)

9. Fu, X., Mei, K.: Approximate controllability of semilinear partial functional differential systems. J. Dyn. Control Syst. 15, 425-443 (2009)

10. Baleanu, D.: Fractional Calculus: Models and Numerical Methods. World Scientific, Boston (2012)

11. Cui, J., Yan, L.: Existence result for fractional neutral stochastic integro-differential equations with infinite delay. J. Phys. A, Math. Theor. 44, 335201 (2011)

12. Kilbas, A.A., Srivastava, H.M., Trujillo, J.J.: Theory and Applications of Fractional Differential Equations. Elsevier, Amsterdam (2006)

13. Lightbourne, J.H., Rankin, S.M.: A partial functional differential equation of Sobolev type. J. Math. Anal. Appl. 93, 328-337 (1983)

14. Revathi, P., Sakthivel, R., Ren, Y.: Stochastic functional differential equations of Sobolev-type with infinite delay. Stat. Probab. Lett. 109, 68-77 (2016)

15. Wang, J., Feckan, M., Zhou, Y.: Controllability of Sobolev type fractional evolution systems. Dyn. Partial Differ. Equ. 11 71-87 (2014)

16. Debbouche, A., Torres, D.F.M.: Sobolev type fractional dynamic equations and optimal multi-integral controls with fractional nonlocal conditions. Fract. Calc. Appl. Anal. 18(1), 95-121 (2014)

17. Benchaabane, A., Sakthivel, R.: Sobolev-type fractional stochastic differential equations with non-Lipschitz coefficients. J. Comput. Appl. Math. 312, 65-73 (2017)

18. Duncan, T.E., Pasikduncan, B., Maslowski, B.: Fractional Brownian motion and stochastic equations in Hilbert spaces. Stoch. Dyn. 2, 225-250 (2002)

19. Biagini, F., Hu, Y., Øksendal, B., Zhang, T.: Stochastic Calculus for Fractional Brownian Motion and Applications. Springer, New York (2008)

20. Mishura, Y.S.: Stochastic Calculus for Fractional Brownian Motion and Related Processes. Springer, Berlin (2008)

21. Feckan, M., Wang, J., Zhou, Y.: Controllability of fractional functional evolution equations of Sobolev type via characteristic solution operators. J. Optim. Theory Appl. 156, 79-95 (2013)

22. Mahmudov, N.I., Denker, A.: On controllability of linear stochastic systems. Int. J. Control 73, 144-151 (2000)

23. Guendouzi, T., Idrissi, S.: Approximate controllability of fractional stochastic functional evolution equations driven by a fractional Brownian motion. ROMAI J. 8(2), 103-117 (2012)

24. Pachpatte, B.G.: Applications of the Leray-Schauder alternative to some Volterra integral and integrodifferential equations. Indian J. Pure Appl. Math. 26, 1161-1168 (1995)

25. Li, Y., Liu, B.: Existence of solution of nonlinear neutral stochastic differential inclusions with infinite delay. Stoch. Anal. Appl. 25, 397-415 (2007)

\section{Submit your manuscript to a SpringerOpen ${ }^{\circ}$ journal and benefit from:}

- Convenient online submission

- Rigorous peer review

- Open access: articles freely available online

- High visibility within the field

- Retaining the copyright to your article

Submit your next manuscript at $>$ springeropen.com 